\title{
Severe bridging fibrosis of the colon in a man with inflammatory bowel disease
}

A 61-year-old man with diabetes and a history of alcohol abuse underwent colonoscopy for the investigation of anal incontinence and urgency of defecation. He had a history of inflammatory bowel disease (ulcerative colitis) and psoriasis from the age of 33 and was on long-term treatment with mesalazine. He had had several severe recurrences of his inflammatory bowel disease that had to be treated with corticosteroids (prednisolone), the last episode occurring at the age of 53 .

Endoscopy of the colon did not reveal any lesions suggestive of a recurrence of the ulcerative colitis, but there was severe bridging fibrosis in the left colon and the sigmoid colon; there were also areas with pseudopolyps and a moderately severe stricture that was difficult to pass with the colonoscope ( Fig. 1). Histological examination of the biopsies only showed some discrete inflammation within the lamina propria ( Fig. 2). The patient was still in remission. Further neurological examination showed the incontinence to be multifactorial, due to neuritis associated with the history of diabetes and alcohol abuse.

Endoscopy with biopsy is an interesting method for the diagnosis and differential diagnosis of inflammatory bowel disease. It is also possible to determine disease activity and the effect and efficacy of medical treatment using this method [1]. In this case we were confronted with a severe bridging fibrosis that had resulted from longstanding ulcerative colitis with several recurrences. Ulcerative colitis is an immunologically mediated disease characterized by chronic inflammation, which results in mucosal damage. Mesenchymal cells are thought to play an important role in the fibrotic process [2].

Endoscopy_UCTN_Code_CCL_1AD_2AD
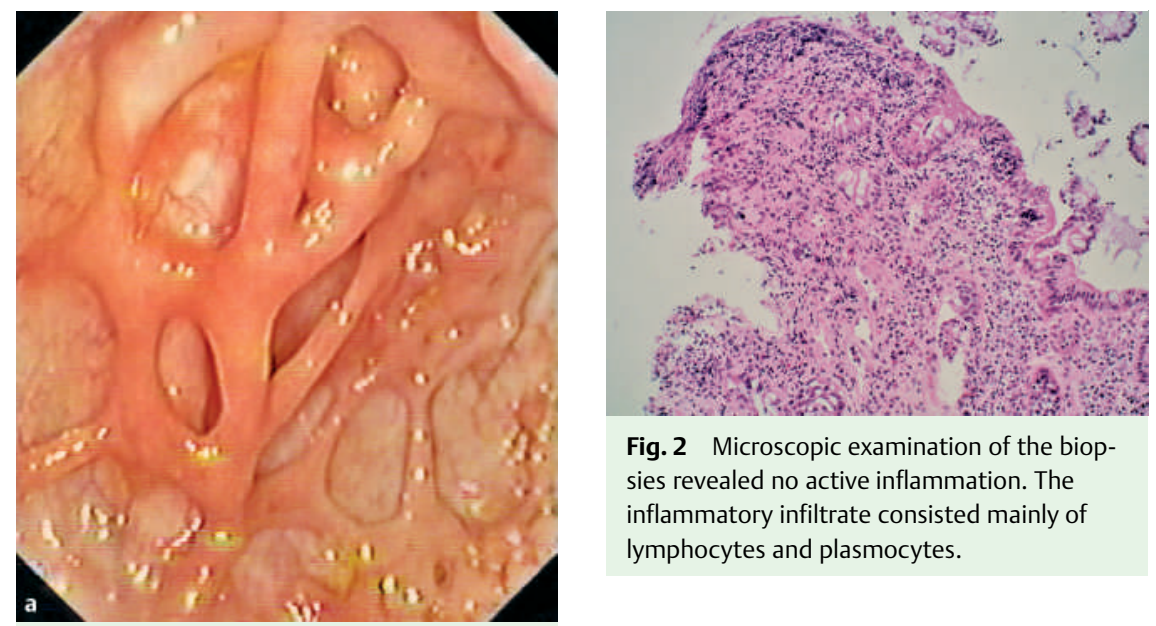

Fig. 2 Microscopic examination of the biopsies revealed no active inflammation. The inflammatory infiltrate consisted mainly of lymphocytes and plasmocytes.

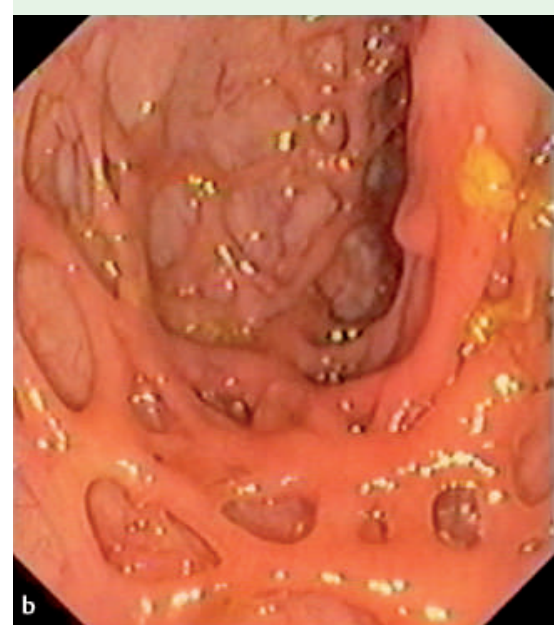

W. Van Moerkercke', G. Deboever ${ }^{2}$,

\section{G. Lambrecht ${ }^{2}$, K. Hertveldt ${ }^{3}$}

Department of Internal Medicine, Gasthuisberg University Hospital, Leuven, Belgium

2 Department of Gastroenterology and Hepatology, AZ Damiaan, Oostende, Belgium

3 Department of Pathology, AZ Damiaan, Oostende, Belgium

\section{References}

1 Geboes K, Dalle I. Influence of treatment on morphological features of mucosal inflammation. Gut 2002; 50: 37-42

2 Pucilowska JB, Williams KL, Lund PK. Fibrogenesis IV. Fibrosis and inflammatory bowel disease: cellular mediators and animal models. Am J Physiol Gastrointest Liver Physiol 2000; 279: G653-G659

\section{Bibliography}

DOI $10.1055 / \mathrm{s}-2007-966418$

Endoscopy 2007; 39: 294

(c) Georg Thieme Verlag KG Stuttgart · New York . ISSN 0013-726X

\section{Corresponding author}

\section{W. Van Moerkercke, MD}

Department of Internal Medicine Gasthuisberg University Hospital Herestraat 49

Leuven

Belgium

Fax: +3216344307

Fig. 1 Colonoscopy showed severe bridging fibrosis in the left colon and the sigmoid colon $(\mathbf{a}, \mathbf{b})$. There were also areas with pseudopolyps and a moderately severe stricture that was difficult to pass with the colonoscope (c). 